Journal of Computer Science 6 (7): 748-755, 2010

ISSN 1549-3636

(C) 2010 Science Publications

\title{
Feature Analysis of Recommender Techniques Employed in the Recommendation Engines
}

\author{
${ }^{1}$ Gopinath Ganapathy and ${ }^{2}$ P.K. Arunesh \\ ${ }^{1}$ Department of Computer Science, Bharathidasan University, Tiruchy-620023, India \\ ${ }^{2}$ Department of Computer Science, Sri SRNM College, Sattur-626203, India
}

\begin{abstract}
Problem statement: Recommender Systems (RS) have become a widely researched area as it is extensively used in web usage mining and E-commerce platforms. Approach: There were a number of recommender systems available to suggest the web pages for the web users. Results: A recommender system acted as an intelligent intermediary that automatically generates and predicts information and web pages, which suit the users' behavior and users' needs. Conclusion: The various recommender models and analyzing the key features of those models and analyzing the features of portal sites that employ recommender systems to help the research community are the key features of this study and survey.
\end{abstract}

Key words: Recommender systems, recommendation engines, estimation methods, extensions to recommender systems

\section{INTRODUCTION}

The challenge of finding the needed information from the web has led to the development of a number of recommender systems, which typically watch the user navigation behavior as a sequence of pages and suggest another set of web pages, products and other information besides the actual information. With the exponential growth of the web, the study of modeling and predicting a user's access on the web has become crucial to the researchers and portal developers.

E-Commerce sites suggest products to their customers by using Recommender systems. Recommended products can be based on the top sellers on a web site, based on the demographics of the customer or the analysis of the past buying behavior of the customer as a prediction for future buying behavior (Schafer et al., 1999). Broadly, these techniques are part of personalization on a site. Loh et al. (2004) is a recommender system that helps travel agents in discovering options for customers, especially those who do not know where to go and what to do. Textual messages transferred between the travel agent and the customers through the private Web chat was analyzed by the system and recommends tourism information. Many on-line stores provide recommendation services for promoting the business are considered.

The research on recommender systems has started as important field as early as 1990 with the appearance of the first study on collaborative filtering. More work has been done in industry, e-commerce and academia on developing new approaches and models for recommender systems over the last decade. The interest and importance in this area still remain very high because it constitutes a problem rich research area. Many tools help users to deal with information overload and provide personalized recommendations and contents. Web usage Mining techniques are used to discover web user profiles and to extract frequent access patterns from the history of the user clickstreams stored in web log files, which can also be used for recommender systems (Nasraoui et al., 2008). Given this backdrop, this study analyzes a set of recommender systems that span the range of different applications and models.

Recommendation approaches: One of the most frequently used representations of recommendation tasks is Collaborative Filtering (CF), essentially a nearest-neighbor method applied to a rating matrix. Collaborative filtering perfectly implements the idea of word-of-mouth promotion, in which the opinions of friends and benchmarking reports could influence predominantly the buying decision. The first applications of recommender technologies were the personalized recommendation of news and Web sites; the former application is often based on collaborative filtering and the latter is based on content-based

Corresponding Author: Gopinath Ganapathy, Department of Computer Science, Bharathidasan University, Tiruchy-620023, India 
filtering. Content-based filtering uses features of items the user liked in the past to infer new recommendations. Unlike collaborative approaches, content-based filtering can't provide serendipitous recommendations. It selects and recommends all products on the basis of purchasing information available from the current user.

In both collaborative and content-based filtering, user profiles are long-term models. These approaches don't exploit deep knowledge about the product domain and both are excellent for supporting the recommendation of simple products such as books or movies. A major strength of these approaches is that no additional knowledge acquisition efforts are necessary if historical data is available (O'Donovan and Smyth, 2005).

In recent years, researchers have proposed more complex statistical models for recommender systems that improve recommendation quality, for example, Bayesian networks with a hidden class variable, compound classification models (Bose et al., 2006) and Relational Markov Models (Anderson et al., 2002). Some have tried hybridization strategies that combine collaborative and content-based information. Meanwhile, others have worked on attribute-aware recommender models that try to take co-user and product information into account in parallel (Loh et al., 2004. Customers purchasing complex products such as financial services, computers, or digital cameras need both information and intelligent interaction mechanisms that support the selection of appropriate solutions. So, an explicit representation of product, marketing and sales knowledge are necessary. Knowledge-based approaches use this type of representation (Krol et al., 2006; Haruechaiyasak et al., 2004; Baraglia et al., 2006). Such deep knowledge lets these recommender systems.

Study on recommender engines: Recommender systems have proven to be effective at delivering the user a more intelligent and proactive information service by making concrete product or service recommendations that are sympathetic to their learned preference and needs by combining ideas from user profiling, information filtering and machine learning (O'Donovan and Smyth, 2005). Recommender systems form a specific type of filtering technique that attempts to present information items, products or services that are likely of interest to the user. Typically, a recommender system compares the user's profile to some reference characteristics and seeks to predict the rating, that a user would give to an item, products or services they have not yet considered. These characteristics may be from the information item, the content-based approach or the user's social environment using the collaborative filtering approach. For example $\mathrm{CF}$ to mobile settings using physical co-presence as the basis for measuring user similarity (De Spindler et al., 2008).

In this study some such recommender systems surveyed and the implemented models, techniques have been studied and presented.

\section{MATERIALS AND METHODS}

Click-stream: Gunduz and Ozsu (2003) proposed a new model based on click-stream tree representation of user behavior. The model considers the order of information pages in a session. The user sessions are clustered based on the similarity of the user sessions. When a request is received from an active user, a recommendation set consisting of three different pages that the user has not yet visited, is proposed using the best matching user sessions. The best matching user session is found by exploring the top $\mathrm{n}$ clusters that have the highest $\mathrm{n}$ similarity values computed using the first two request of the active user session. The rest of the recommendations for the same active user session are made by using the top $n$ clusters. The novelty of the approach lies in the method of computing the similarity of user sessions and the clustering. Session similarity measure is based on FastLSA sequence alignment method. The clustering of user sessions reduces the search space and each cluster is represented by a clickstream tree. The model recommends three pages when the time of the last visited page of the active user sessions are given. The click-stream tree enables to insert the entire session of the user with out any information loss. They considered the problem of modeling the behavior of a web user during a single visit to the web site.

Concept hierarchy: Bose et al. (2006) proposed another model that effectively combines usage information with information from the conceptual structure of the web site to generate recommendations. It is important to note that the hierarchy of pages of an individual web site is considered, not deal with a hierarchy of the entire web. The prediction model is using click-stream trees. Each node in the tree consists of page identifier, time spent and a counter for sessions traversed in each node. Users session is matched against the sessions in the clusters and recommendation score is assigned to each such match. Recommendations are given based on the highest score to the user. 
With time impact coefficient: Krol et al. (2006) proposed two generic recommender mechanisms, implemented in cadastre internet information system. List of last queries submitted by the user and list of page profiles recommended to a user are the two systems proposed. The page recommendation is based on the concept of the page profile which represents the system option, type of retrieval mechanisms and search criteria. The rank values for the page profiles are calculated based on the usage frequency and the time impact coefficient. Rank value $r_{u}\left(p_{j}\right)$ of a page profile $p_{j}$ used by user $u$ is calculated in the following way:

$$
\mathrm{r}_{\mathrm{u}}\left(\mathrm{p}_{\mathrm{j}}\right)=\sum_{\mathrm{d}=0}^{\mathrm{Du}-1} \mathrm{f}_{\mathrm{ud}}\left(\mathrm{p}_{\mathrm{j}}\right) *\left(1-\frac{\mathrm{d}}{\mathrm{D}_{\mathrm{u}}}\right)
$$

where, $f_{u d}\left(p_{j}\right)$ is the usage frequency of page profile $p_{j}$ by $\mathrm{u}$ and $\mathrm{d}$ days back before the day of rank calculation. The recommended page is selected by the user from the list facilitates and the search.

Web recommender system: Haruechaiyasak et al. (2004) proposed a new framework based on data mining algorithms, combines both content-based and collaborative filtering methods. Content-based filtering integrates both textual analysis and the user personalization during recommendation process, collaborative filtering is based on the method of mining user access patterns. This approach has a better potential of reducing the user access time on the web site compared to the existing Markov model approach. The response time of this system is very high. This proposed framework is applied to construct a system prototype for recommending the University of Miami's web pages and automatically generates recommended list of web pages based on users preferences.

$\pi$ Suggest: Baraglia et al. (2006) contributed a privacy enhanced RS $\pi$ Suggest, that allows for creating serendipity recommendations with out breaching users privacy. The system that helps users to navigate through the web by providing dynamically generated links to pages that have not yet been visited and are of potential interest. The architecture of $\pi$ Suggest is based on a two tier structure. One of them is the browser at the client-side. The other tier is based on an incremental personalization procedure, tightly coupled with the Web server. Its knowledge base is incrementally updated by monitoring usage data and then notified to the client, which will be able to use it to personalize onthe-fly the requested HTML page, by appending a list of page links. This system does not maintain any database, it needs to maintain a data structure i.e., an undirected graph from which recommendations were made. A triangular adjacency matrix is used to store the knowledge base. When a session starts on clients side, the $\pi$ Suggest plug in asks the server for page clusters extracted from the knowledge base. The plug in holds the user session and creates the suggestions for the user.

ICPF: Zhu et al. (2003) describes an effective complete-web recommender system. Information content pages ICPF, This system is in client side and it is not specific to a single website but can point users to pages anywhere in the web. The goal of this approach is to recommend only "Information Content" (IC) pages; i.e., pages that are essential to the user's task. To determine this, first a set of annotated web logs were collected, from which the learning algorithm learned to characterize the IC-page associated with the pages in any partial sub sessions contribution. IC-pages for the users are estimates from the current click-stream, based on properties of the words that appear, that is, if the word $\mathrm{w}$ appears in at least 2 recent pages and $\mathrm{w}$ appears in a snippet that was followed, then $\mathrm{w}$ will tend to appear in IC-pages. This rule assert the IC-word and ICPF compute the browsing properties for essential of all the words that appear in any of the pages and this model is used to predict the users current information need.

AIE is used as a client-side tool for collecting the training data, which allows the user to explicitly indicate which pages were IC-pages for the specific current task. ICPF system needs client-side information in the form of annotated web-logs, the sequence of web pages that users have visited. The pages are separated into a sequence of "IC-sessions" and ends with an ICpages or the end of the users entire session.

Recommendation method for LTV: Iwata et al. (2008) present a novel recommendation method that maximizes the probability of the Lifetime Value (LTV) for the customers. The method finds frequent purchase patterns among high LTV users and recommends items for a new user that simulate the found patterns (Iwata et al., 2008).

Survival analysis techniques were used and efficiently find the patterns from the log data. Users interests are inferred from the purchase history and based on the interests the effective recommendations were generated. To estimate users interest, the maximum entropy models was used. To increase LTV, online stores providing measured services and encourage users to purchase many items. On the other hand, online stores providing subscription services need to encourage users to extend their subscription periods. Therefore, online stores should recommend items using 
different strategies depending on measured or subscription services and the authors proposed different recommendation methods for each of them. The method recommends item $\breve{s}$ that maximizes $\mathrm{P}(\mathrm{l} \mid \mathrm{u}, \mathrm{s})$, which is the probability of improving the LTV of user $u$ when item $\mathrm{s}$ is recommended, as follows:

$$
\check{\mathrm{S}}=\underset{\mathrm{s} \in \mathrm{S}}{\arg \max } \mathrm{P}(1 \mid \mathrm{u}, \mathrm{s})
$$

Where:

$\mathrm{s}=\mathrm{A}$ set of items

$1=$ The improvement of the LTV

$\mathrm{S}=$ The recommended item

and also recommended $\mathrm{m}$ items with the highest $\mathrm{P}(\mathrm{l} \mid \mathrm{u}, \mathrm{s})$ values.

\section{RESULTS AND DISCUSSION}

Implementation of recommended systems in web: The RS compares the collected data to similar information collected from others and calculates a list of recommended items for the user. Commercial and non-commercial examples are identified in this study. Recommender systems are a potential alternative to search algorithms. Since RS help users discover items that they might not have found by themselves. Interestingly enough, recommender systems are often implemented using search engines indexing nontraditional data (Wikipedia, 2009a). Here we present some of the businesses that utilize recommender system technology in their web sites (Wikipedia, 2009b)

Amazon.com allows users to submit reviews to the web page of each product. As part of their review, users must rate the products. Rating Scale was given in the site that is from one to five stars. ChoiceStream is one of the sites that offer pay for performance marketing services. Collarity is a privately funded web services that provide a recommendation system, social search and online advertising services for web publishers. Digg-a social news website, eBay.com, an online auction and shopping website, Heeii, IMDb, Last.fm, LibraryThing, StumbleUpon, Amie Street-an online music store and social network service, iLike-an online service site allows users to download and share music, iTunes-a proprietary digital media player application, used for playing and organizing digital music and video files and Musicmatch are some other E-commerce sites that provide recommendations using collaborative filtering (Wikipedia, 2009a; 2009b)
Netflix.com is another online DVD rental service site which maintains an extensive personalized videorecommendation system based on ratings and reviews by its customers, similar to the system used by Amazon.com, Netflix uses RS to suggest movies to its 10 million-plus customers and they challenged the developers communities to beat the accuracy of their RS (Monroe, 2009; Ampazis, 2008). In Table 1 it is attempted to summarize the business applications, recommendation technology, interfaces and findings that is how the users find recommendations for all the example and applications.

Survey of the recommender engines and techniques: The Recommended methods described in this study, are good in several applications, including the ones for real estate cadastre web site RS (Krol et al., 2006), social network Mobile setting (De Spindler et al., 2008), recommending books (Kawashoma et al., 2006), news articles, pages in NASA and Clark net sites (Gunduz and Ozsu, 2003), web server logs of an college, tourism (Loh et al., 2004) movies (Ampazis, 2008), health care system and other items discussed in this study, Implementation of RS in Web. All the businesses described in implementation of RS in Web and (Gunduz and Ozsu, 2003; Kawashoma et al., 2006) were based on the model, collaborative filtering.

Recommendation interface and findings are the two more criteria's considered for this survey. Through the examples of recommender system, we have identified different methods for finding recommendations each of which may provide access to more than one recommendation interface and/or technology. In finding Selection option process, the customers must interact with the system in order to receive the recommendations. The Predefined options are the base for their recommendations. For example Amazon.com has more predefined choices.

In Table 2 it is attempted to summarize the features of recommendation technology. The models and technology used and proposed, view of data/main data, representation of data, methods used, the application categories and algorithms used are considered for the feature analysis. The RS described in study on Recommender Engines is summarized in Table 2 that can be extended to the capabilities of RS (Adomavicius and Tuzhith, 2005), that includes understanding of users and items, model-based recommendation techniques, multidimensional views of recommendations, multi criteria ratings, nonintrusiveness, flexibility, effectiveness, trustworthiness, scalability and privacy issues of RS. 
Table 1: Recommendation features implemented in portals

\begin{tabular}{|c|c|c|c|}
\hline Applications & $\begin{array}{l}\text { Model based recommended } \\
\text { techniques used }\end{array}$ & $\begin{array}{l}\text { Recommendation } \\
\text { interface }\end{array}$ & Finding Recommendations \\
\hline Amazon.COM & Collaborative filtering & Rating Scale & Selection options organic navigation \\
\hline BAYNOTE & Active filtering, passive filtering & Nonintrusive & $\begin{array}{l}\text { Based on previous visitors with similar interests in } \\
\text { similar contexts }\end{array}$ \\
\hline ChoiceStream & Collaborative filtering cohort analysis & Automated and personalized rating & Off base suggestions using multiple correlation tables \\
\hline Collarity & Collaborative filtering & Nonintrusive implicit & Based on information value hierarchy \\
\hline Digg & Collaborative filtering & Nonintrusive implicit & Voting basis \\
\hline eBay INC. & Passive filtering & Implicit behavior content based & Organic Navigation \\
\hline Heeii & Passive filtering $\mathrm{CF}$ & Nonintrusive & Based on array of implicit behavior \\
\hline $\begin{array}{l}\text { IMDb- internet } \\
\text { movie database }\end{array}$ & $\mathrm{CF}$ & Rating scale intrusive & Explicit ratings \\
\hline LAST.FM & $\mathrm{CF}$ & Explicit feedback intrusive & Based on Multi criteria ratings \\
\hline LibraryThing & Collaborative filtering & Implicit knowledge & Based on catalog with similar data \\
\hline Strands, INC. & Collaborative filtering & $\begin{array}{l}\text { History of other members, } \\
\text { intrusiveness }\end{array}$ & Find other members with similar tastes to their own \\
\hline Netfix & $\mathrm{CF}$ & Intrusive real ratings & Based on Explicit ratings \\
\hline StumbleUpon & Collaborative filtering & Multi criteria ratings & Explicit rating \\
\hline
\end{tabular}

Table 2: Recommendation system feature chart

\begin{tabular}{|c|c|c|c|c|c|c|}
\hline $\begin{array}{l}\text { Recommender } \\
\text { systems }\end{array}$ & $\begin{array}{l}\text { Model, technology } \\
\text { used and proposed }\end{array}$ & $\begin{array}{l}\text { View of data/ } \\
\text { main data }\end{array}$ & Representation & Method & Application categories & Algorithms used \\
\hline$\overline{1}$ & $\begin{array}{l}\text { Two-dimensional } \\
\text { sequential model }\end{array}$ & $\begin{array}{l}\text { Server logs NASA and } \\
\text { Clark net web server }\end{array}$ & $\begin{array}{l}\text { Graph click-stream } \\
\text { tree }\end{array}$ & $\begin{array}{l}\text { FastLSA sequence } \\
\text { alignment method }\end{array}$ & $\begin{array}{l}\text { Calculating the similarity } \\
\text { of user sessions, identical } \\
\text { pages and time spent on } \\
\text { pages, graph based } \\
\text { clustering }\end{array}$ & $\begin{array}{l}\text { Build click-stream tree } \\
\text { algorithm, find best path } \\
\text { algorithm }\end{array}$ \\
\hline 2 & $\begin{array}{l}\text { Concept hierarchy, } \\
\text { prediction model using } \\
\text { click-stream tree, } \\
\text { similarity model method }\end{array}$ & $\begin{array}{l}\text { Session similarity } \\
\text { Matrix, web } \\
\text { server logs }\end{array}$ & $\begin{array}{l}\text { Click-stream } \\
\text { tree graph }\end{array}$ & $\begin{array}{l}\text { Sequence alignment } \\
\text { concept aware } \\
\text { similarity model }\end{array}$ & $\begin{array}{l}\text { Similarity score, sessions } \\
\text { are clustered, click } \\
\text { stream sessions }\end{array}$ & $\begin{array}{l}\text { Cluto-clustering algorithm } \\
\text { based on graph partitioning } \\
\text { build click stream tree }\end{array}$ \\
\hline 3 & $\begin{array}{l}\text { Search criteria and } \\
\text { option tree with } \\
\text { search forms }\end{array}$ & $\begin{array}{l}\text { Log of submitted } \\
\text { queries, log of } \\
\text { recommended pages }\end{array}$ & $\begin{array}{l}\text { Rank value of } \\
\text { pages }\end{array}$ & Page profile & $\begin{array}{l}\text { Page profiles } \\
\text { recommended to the user, } \\
\text { list facilitate and search }\end{array}$ & $\begin{array}{l}\text { Query criteria, rank value, } \\
\text { page profile recommendation }\end{array}$ \\
\hline 4 & $\begin{array}{l}\text { Combines collaborative } \\
\text { filtering and content } \\
\text { based utilizes data } \\
\text { mining techniques }\end{array}$ & $\begin{array}{l}\text { URL's, log records, } \\
\text { Crawling and } \\
\text { parsing textual and } \\
\text { hyperlink contents. }\end{array}$ & $\begin{array}{l}\text { Page similarity } \\
\text { in the keywords, }\end{array}$ & $\begin{array}{l}\text { Association rule } \\
\text { mining }\end{array}$ & $\begin{array}{l}\text { Integrates textual analysis } \\
\text { and user personalization, } \\
\text { University web site } \\
\text { focused }\end{array}$ & $\begin{array}{l}\text { SQL queries, association } \\
\text { rules }\end{array}$ \\
\hline 5 & Classification & $\begin{array}{l}\text { Triangular adjacency } \\
\text { matrix }\end{array}$ & Undirected graph & $\begin{array}{l}\pi \text { suggest, a } \\
\text { privacy enhanced } \\
\text { recommender system }\end{array}$ & $\begin{array}{l}\text { User ratings considered, } \\
\text { two tier system work both } \\
\text { in server and client side }\end{array}$ & $\begin{array}{l}\text { Incremental connected } \\
\text { components algorithm }\end{array}$ \\
\hline 6 & $\begin{array}{l}\text { Naïve Bays (NB) } \\
\text { classifier }\end{array}$ & $\begin{array}{l}\text { Set of annotated web } \\
\text { logs, IC labels in each } \\
\text { page }\end{array}$ & Information pages & $\begin{array}{l}\text { Sequential attributes, } \\
\text { search query }\end{array}$ & $\begin{array}{l}\text { Not for a single site, } \\
\text { can point users to pages } \\
\text { anywhere in the web }\end{array}$ & $\begin{array}{l}\text { AIE Browser (Annotation } \\
\text { Internet Explorer) ICSI }\end{array}$ \\
\hline 7 & $\begin{array}{l}\text { Maximum entropy } \\
\text { model for estimate } \\
\text { user's interest, life } \\
\text { time value, frequency } \\
\text { model, collaborative } \\
\text { filtering }\end{array}$ & $\begin{array}{l}\text { Log data, user } \\
\text { purchase history }\end{array}$ & ---- & Survival analysis & $\begin{array}{l}\text { Probability }(\mathrm{Q}, \mathrm{R}, \mathrm{NO} \\
\text { recommend) }\end{array}$ & $\begin{array}{l}\text { User behavior in a measured } \\
\text { service and subscription } \\
\text { service }\end{array}$ \\
\hline
\end{tabular}

Most of the recommender systems produce ratings that are based on a limited understanding of the users. The RS methods produce ratings that are based on the understanding of the users and items as captured by the user and item profiles. The methods take full advantage of the information in the user's transactional histories and other available data. Classical collaborative filtering methods use the user and item profiles for recommendation purpose and rely exclusively on the rating information to make recommendations.

Using various statistical and machine learning techniques, some of the model-based approaches provide rigorous rating estimations. Other areas of mathematics and computer science, like mathematical approximation theory, Memory-based and model based $\mathrm{CF}$ algorithms predict recommendation value based on the rating matrix (Su et al., 2007; Zhu et al., 2005; Shai et al., 2005) also contribute to developing better rating estimation methods and machine learning models. For example MDP is a model for sequential stochastic decision problem (Shai et al., 2005). Drachsler et al. (2008) Proposed memory-based recommender system techniques.

Recommender systems are proposed to work in multidimensional settings. Two dimensional userx items space were used in earlier RS. That is the recommendations were made based only on the user and item information and do not take into consideration the 
additional contextual information. Some of the applications may be critical because the utility of a certain product to a user may depend on time (Iwata et al., 2008; Petridou et al., 2008). Time of the year, such as season, month, day or week and it also depends on the persons be whom the product will be consumed or shared and under which circumstances. Time-related issues in web users, the duration of the user's accessing on a page and the succession of their visits i.e., click-streams (Bose et al., 2006; Gunduz and Ozsu, 2003), in such situations, the RS must take additional contextual information. Such as time, place, company and other related information of user, into consideration when recommending a product or other information. So the system is in need of multidimensional settings. The inclusion of the knowledge about the user's task into the RS algorithm leads better recommendation (Petridou et al., 2008; Adomavicius and Tuzhilin, 2008). RS mostly deals with single criterion ratings, such as ratings of movies, songs, books and other products, However, in some applications, such as restaurant recommendations and tourism recommendations (Loh et al., 2004).

Recent studies have indicated that multiple item characteristics may be taken into consideration when making a recommendation. It might not be sufficient to base recommendation on single-attribute ratings. So the researchers highlighted the potential need of MultiCriteria Decision Making methods in recommender systems (Manouselisi, 2008). Incorporate Multi-criteria ratings into recommendation methods, finding optimal solutions, taking linear combinations of multiple criteria and reducing the problem to a single-criteria optimization problem, optimizing the most important criterion and converting other criteria constraints are some of the methods used in recommender systems for multi-criteria ratings.

Most of the recommender systems are intrusive in the sense that they require explicit feedback from the user and often at a significant level of user involvement. For example, before recommending any products, movies, books or news group articles, the system needs to acquire the ratings of previously read books or articles and, often many of them. Some of the RS uses nonintrusive rating determination methods where certain proxies are used to estimate real ratings. For example, the amount of time a user spends time for reading an article, book or web site can serve as a proxy of the site or article's rating given by the user (Adomavicius and Tuzhith, 2005; Zhu et al., 2002).

Flexibility, effectiveness of recommendation as speed address based on metrics, Scalability and privacy are some of the issues and features of recommender system. RS also underlines many less overtly commercial sites, such as those providing music or news. But in each case a recommender system tries to discern a user's likely preferences from a frustratingly small data set about that user (Monroe, 2009). Web based RS is very popular nowadays. Further, critical challenge for Web-based recommender systems is generating accurate results in less than a second. To maintain rapid responses as databases grow, researchers want continually trade off effectively with speed, accuracy, privacy and security.

RS is technology based system and provides personalized recommendations to the web users. Recommendations are suggested from opinions and actions of other users with similar tastes. However, with increasing popularity of recommender systems in Ecommerce sites they have become susceptible to shilling attacks. In shilling attacks, attackers try to influence the system by inserting biased data into the system. An attack on a RS is mounted by injecting a set of biased attack profiles into the system. Each attack profile contains biased rating data and a target item. Profiles are injected into the system by fictitious user identities created by the attacker. Every attack can be classified as a push attack or a nuke attack. In a push attack, the objective of the attacker is to increase the likelihood of the target item being recommended to a large section of the users in the system. While in a nuke attack, the objective is to prevent the target item from being recommended (Ray and Mahanti, 2008). So new algorithms needed to improve robustness and stability of recommender systems against such security problems.

\section{CONCLUSION}

RS has become more or less the backbone of many of the major firms and businesses on the Web space. The next generation of RS will rely more on implicit information, such as the pages or items that a web user clicks on while navigating a web site. Based on the users navigation patterns, the system correlating the products or pages and the user's will get very valuable information as suggested by the RS.

In this study, some of the recommendation system technologies and application examples were surveyed. The field of recommendation system is still young and much work lies ahead. Given the huge amount of information available on the Internet and increasingly important role that the Web plays in today's society, Web usage mining services will become the most flourishing subfields in Web mining. However the contemporary models of recommender system surveyed 
in this study still require further research in security and privacy to be applied in a wider range of applications. Also the evaluation of RS models is a challenging as there is no concrete framework or base template for analysis.

\section{REFERENCES}

Adomavicius, G. and A. Tuzhith, 2005. Toward the next generation of recommender system: A survey of the state-of-art and possible extensions. IEEE Trans. Knowl. Data Eng., 17: 734-749.

Adomavicius, G. and A. Tuzhilin, 2008. Extending recommender systems: A multidimensional approach. Proceedings of the International Joint Conference on Artificial Intelligence, (IJCAI'08), pp: $1-5$.

Ampazis, N., 2008. Collaborative filtering via concept decomposition on the netflix dataset. Proceedings of the ECAI2008 Workshop, pp: 1-5. http://www.feeds2.com/netflix/Ampazis_ECAI08.pdf

Anderson, C.R., P. Domingos and D.S. Weld, 2002. Relational Markov models and their application to adaptive web navigation. Proceedings of the 8th ACM SIGKDD International Conference on Knowledge Discovery and Data Mining, July 2326, ACM Press, Edmonton, Alberta, Canada, pp: 143-152. http://portal.acm.org/citation.cfm?id=775047.775068

Baraglia, R., C. Lucchese, S. Orlando, M. Serrano' and F. Silvestri, 2006. A privacy preserving recommender system. Proceedings of the 2006 ACM Symposium on Applied Computing, Apr. 2327, ACM Press, Dijon, France, pp: 559-563. http://portal.acm.org/citation.cfm?id=1141277.1141407

Bose, A., K. Beemanapalli, J. Srivastava and S. Sahar, 2006. Incorporating concept hierarchies into usage mining based recommendations. Proceedings of the 8th Knowledge Discovery on the Web International Conference on Advances in Web Mining and Web Usage Analysis, ACM Press, Philadelphia, PA, USA., pp: 110-126. http://portal.acm.org/citation.cfm?id=1784822

De Spindler, A., S. Leone, M. Grossniklaus and M.C. Norrie, 2008. Harnessing facebook for the evaluation of recommender systems based on physical copresence. Proceedings of the ECAI2008 Workshop on Recommender Systems, Patras, Greece, pp: 1-6.

Drachsler, H., G.K. Hummel and R. Koper, 2008. Recommendations for learners are different; Applying memory-based recommender system techniques to lifelong learning. Int. J. Learn. Technol., 3: 404-423.
Gunduz, S. and M.T. Ozsu, 2003. A web page prediction model based on click-stream tree representation of user behavior. Proceedings of the 9th ACM SIGKDD International Conference on Knowledge Discovery and Data Mining, Aug. 2427, ACM Press, Washington DC., pp: 535-540. http://portal.acm.org/citation.cfm?id=956815

Haruechaiyasak, C., M.L. Shyu and S.C. Chen, 2004. A data mining framework for building a web-page recommender system. Proceedings of the 2004 IEEE International Conference on Information Reuse and Integration, Nov. 8-10, IEEE Xplore Press, USA., pp: 357-362. DOI: 10.1109/IRI.2004.1431487

Iwata, T., K. Saito and T. Yamada, 2008. Recommendation method for improving customer lifetime value. IEEE Trans. Knowl. Data Eng., 20: 1254-1263. DOI: 10.1109/TKDE.2008.55

Kawashoma, H., T. Matsushita and S. Satake, 2006. PORSCHE: A physical objects recommender system for cell phone users. Proceedings of the 2nd International Workshop on Personalized Context Modeling and Management for UbiComp Applications, Sept. 18-18, California, USA., pp: 1-5. http://www.ayu.ics.keio.ac.jp/orien/2008/research/a chieve/pdf/2006/satake_Paper7.pdf

Krol, D., M. Szymanski and B. Trawinski, 2006. The recommendation mechanism in an internet information system with time impact coefficient. Int. J. Comput. Sci. Appli., 3: 65-80.

Loh, S., F. Lorenz, R. Saldana and D. Licthnow, 2004. A tourism recommender system based on collaboration and text analysis. Inform. Technol. Tourism, 6: 157-165.

Manouselisi, N., 2008. A discussion on multi-criteria recommendation. Proceedings of the ECAI2008 Workshop on Recommender Systems, Patras, Greece, pp: 64-69.

Monroe, D., 2009. Recommender system news just for you. Commun. ACM. 2: 15-17.

Nasraoui, O., M. Soliman, E. Saka, A. Badia and R. Germain, 2008. A web usage mining framework for mining evolving user profile in dynamic web sites. IEEE Trans. Knowl. Data Eng., 20: 202-205. http://portal.acm.org/citation.cfm?id=1340194

O'Donovan, J. and B. Smyth, 2005. Trust in recommender systems. Proceedings of the IUI'05, Jan. 9-12, ACM Press, San Diego, California, USA., $\quad$ pp: 167-174. http://www.csi.ucd.ie/UserFiles/publications/11253 25051774.pdf

Petridou, S.G., V.A. Koutsonikola, A.I. Vakali and G.I. Papadimitriou, 2008. Time aware web users clustering. IEEE Trans. Knowl. Data Eng., 20: 653-667. 
Ray, S. and A. Mahanti, 2008. Filler items strategies for effective shilling attacks. Proceedings of the ECAI2008 Workshop on Recommender Systems, Patras, Greece, pp: 38-40.

Schafer, J.B., J. Konstan and J. Riedl, 1999. Recommender systems in e-commerce. Proceedings of the 1st ACM Conference on Electronic Commerce, Nov. 3-5, ACM Press, Denver, Colorado, United States, pp: 158-166. http://portal.acm.org/citation.cfm?id=337035

Shai, G., D. Heckerman and R.I. Brafman, 2005. An MDP-based recommender system. J. Mach. Learn. Res., $6:$

1265-1295. http://jmlr.csail.mit.edu/papers/v6/shani05a.html

$\mathrm{Su}$, X., R. Greiner, T.M. Khoshgoftaar and X. Zhu, 2007. Hybrid collaborative filtering algorithms using a mixture of experts. Proceedings of the IEEE/WIC/ACM International Conference on Web Intelligence, Nov. 2-5, IEEE Computer Society, Washington DC., USA., pp: 645-649. http://portal.acm.org/citation.cfm?id=1331740.133 1782
Wikipedia,

2009a.

Collaboration. http://en.wikipedia.org/wiki/Collaboration

Wikipedia, 2009b. Recommendation. http://www.wikipedialrecommender_systems

Zhu, T., R. Greiner and G. Haubl, 2002. Important page predicting for internet recommendation: A machine learning way. Proceedings of the International Conference on Fuzzy Systems and Knowledge Discovery, Nov. 2002, Singapore.

Zhu, T., R. Greiner and G. Haubl, 2003. An effective complete-web recommender system. http://www2003.org/cdrom/papers/alternate/P718/p 718-tszhu.html

Zhu, T., R. Greiner, G. Haubl, K. Jewell and B. Price, 2005. Using learned browsing behavior models to recommend relevant web pages. Proceedings of the 19th International Joint Conference on Artificial Intelligence, July 30-Aug. 5, ACM Press, Edinburgh, Scotland, pp: 1589-1590. http://portal.acm.org/citation.cfm?id=1642293.164 2569 